\title{
Transcriptomic Analysis Reveals a Link Between Hippo Signaling Pathway and Macrophages in Lungs of Mice with OVA-Induced Allergic Asthma
}

\author{
Huan Xiao $\mathbb{D}^{\prime}$, Qian-nan Zhang', Qi-xiang Sun $\mathbb{D}^{2}$, Lao-dong Li $\mathbb{D}^{2}$, Si-yue Xu², Chao-qian Li $\mathbb{D}^{\prime}$ \\ 'Department of Emergency, The First Affiliated Hospital of Guangxi Medical University, Nanning, 53002I, Guangxi Province, People's Republic of \\ China; ${ }^{2}$ Department of Respiratory Medicine, The First Affiliated Hospital of Guangxi Medical University, Nanning, 53002I, Guangxi Province, \\ People's Republic of China
}

Correspondence: Chao-qian Li, Department of Emergency, The First Affiliated Hospital of Guangxi Medical University, 6 Shuangyong Road, Nanning, 53002I, Guangxi Province, People's Republic of China, Tel +86 I3807887867, Fax +86 77I-535003I, Email Lichaogiangood@I63.com

\begin{abstract}
Purpose: The Hippo signaling pathway participates in the restriction of cell proliferation and organ growth. Activated macrophages have been implicated in the pathogenesis of allergic asthma. Recent studies have shown that Hippo signaling pathway may also be involved in the regulation of asthma. However, the link between Hippo signaling pathway and macrophages in the context of allergic asthma has not been investigated. The purpose of this study was to explore the link between Hippo signaling pathway and macrophages using a mice model of OVA-induced allergic asthma.

Methods: Mice models of asthma were established. Lung tissues were collected from mice and pooled for mRNA sequencing and bioinformatics analysis. The relative mRNA expression of Hippo signalling pathway-related proteins Yap1, Lef1 and Ctgf was also measured. Double immunofluorescence staining was performed on lung tissues to evaluate macrophage marker F4/80 expression and Yap1/Lef1/Ctgf expression.

Results: Results of the RNA-Seq of lung tissues demonstrated that the Hippo signaling pathway was down-regulated in OVA-induced allergic asthma. Using the cytoHubba tool kits in Cytoscape, the following top 10 hub genes of Hippo signalling pathway were identified: Yap1, Lef1, Ctgf, Ccnd1, Axin2, Smad7, Wnt4, Wnt3a, Pard6b, and Wwc1. Using the seq-ImmuCC (http://218.4.234.74:3200/immune/), a negative correlation was found between macrophages and Hippo signaling pathway activity $\left(\mathrm{R}^{2}=0.93\right)$. The mRNA expression levels of pulmonary Yap1, Lef1, and Ctgf were down-regulated in the mice model of OVA-induced allergic asthma. Moreover, double-stained immunofluorescence for F4/80 and Yap1, Lef1, Ctgf in mouse lung sections respectively revealed that macrophage proliferation was correlated with downregulation of the Hippo signaling pathway in the mice model of OVA-induced allergic asthma.

Conclusion: These results demonstrated that the Hippo signaling pathway was down-regulated in asthma mice, and the proliferation of macrophages was associated with downregulation of the Hippo signaling pathway. These findings reveal novel insights into the pathogenesis and treatment of asthma.
\end{abstract}

Keywords: Hippo signaling pathway, asthma, macrophages, epithelial cells

\section{Introduction}

Asthma is a global chronic respiratory disease characterized by airway inflammation, airway hyper-responsiveness (AHR), reversible airflow obstruction, airway remodeling, eosinophilic infiltration, mucus overproduction and goblet cell hyperplasia. ${ }^{1}$ It is a complex, heterogeneous disease affecting more than 350 million people worldwide. It is also a significant cause of increased mortality and decreased quality of life. Currently, asthma is commonly treated using glucocorticoids. However, the use of glucocorticoids is limited due to its associated drug resistance and adverse side effects. In addition, this method negatively affects bone mineral density and growth of children. ${ }^{2}$ Therefore, it is crucial to understand the underlying molecular pathogenesis of asthma and to explore other effective targeted therapies. 
The Hippo signaling pathway was initially discovered in drosophila as a conserved signal transduction pathway. Several studies have shown that the Hippo signaling pathway regulates growth and organ size by inhibiting cell proliferation as well as promoting cell apoptosis. ${ }^{3,4}$ The key regulatory mechanisms of the Hippo signaling pathway are a series of kinase cascades driven by MST1/2 kinases (Orthologs of Drosophila Hippo) and their downstream kinases LATS1/2 in mammals. Yap and its paralog Taz are the two key downstream effectors of the Hippo signaling pathway. Hippo signaling pathway suppresses Yap/Taz transcription activities by activating Lats kinases that directly phosphorylate Yap/Taz. This facilitates cytoplasmic retention and subsequent degradation of Yap/Taz. The unphosphorylated Yap/ Taz translocates into the nucleus to bind to the transcription enhancer factor (TEAD/TEF) family of transcriptional factors, thus promoting cell growth and proliferation. ${ }^{5,6}$

Previous studies have revealed that the Hippo signaling pathway is relevant to cardiac regeneration, ${ }^{7}$ tumorigenesis, ${ }^{8}$ stem cell biology ${ }^{9}$ and can respond to ${ }^{10}$ and mediate ${ }^{3}$ inflammatory signals. Recent studies have found that Hippo signaling pathway plays an important role in immune function, including innate immunity and autoimmunity. ${ }^{11}$ Allergic asthma is a pulmonary inflammatory disorder in which immune imbalances are central to the pathogenesis. ${ }^{12}$ Therefore, this study speculated that the Hippo signaling pathway plays an important role in immunomodulatory processes in asthma pathogenesis.

It is known that asthma exacerbations are associated with abnormal immune proliferation. Macrophages are specialized antigen presenting cells that have important functions in innate defense against infection, in clearance of host infected cells and molecules and in viral antigen presentation. ${ }^{13}$ Activated macrophages release pro-inflammatory mediators that play a major role in the pathogenesis of asthma. These include NO, reactive oxygen species, interleukin$1 \beta$ and tumor necrosis factor- $\alpha$ among other inflammatory mediators. A recent study demonstrated that liver injury in mice and humans increases levels of YAP/TAZ/CYR61 in hepatocytes, thus attracting macrophages to the liver, which promote inflammation and fibrosis. ${ }^{14}$ However, the mechanisms linking macrophages and Hippo signaling pathway in asthma are unknown. In the present study, we aim to explore the link between macrophage proliferation and Hippo signaling pathway using a mice model of OVA-induced allergic asthma.

\section{Materials and Methods}

\section{Experimental Animals}

Balb/c mice (male, between 4 and 6 weeks) were acquired from Changsha Tianqin Biotechnology Co., Ltd (License number: SCXK (Xiang) 2019-0014, Changsha, China). The mice were housed under approved specific pathogen-free laboratory conditions. Furthermore, the protocols used in this study were approved by Guangxi Medical University Ethics Committee (Approval No.202103006). The experimental protocols strictly followed the 'Guiding Principles for the Use and Care of Experimental Animals issued by the Ministry of Science and Technology of China. The total number of animals and their suffering was minimized according to the $3 \mathrm{R}$ principle.

\section{Induction of Allergic Asthma Mice Model}

Balb/c mice were randomly assigned into two groups ( $\mathrm{n}=6 \mathrm{mice} /$ group): normal control group (NC) and asthma model group (OVA-induced mice). The asthma model was based on our previous study. ${ }^{15}$

Each mouse was intraperitoneally injected with an OVA (Grade V, Sigma, St. Louis, MO, USA) mixture (25 $\mu$ g OVA, $1 \mathrm{mg}$ aluminum hydroxide adjuvant and $0.2 \mathrm{~mL}$ PBS) on days 0,7 and 14 for sensitization. From days 21 to 28 , the mice were challenged with $2 \%$ OVA using an ultrasonic nebulizer (WH-2000, Guangzhou, China) in a closed chamber $(20 \times$ $30 \times 20 \mathrm{~cm}^{3}$ ) for 30 minutes (Figure 1A).

\section{Measurement of Airway Responsiveness}

Airway responsiveness was determined using a noninvasive lung function machine (Fine-Pointe ${ }^{\mathrm{TM}}$ NAM system TBL4500, Buxco, Wilmington, NC) after the final challenge. The airway responsiveness was measured through specific airway resistance (sRaw), and the detection method was referenced from our previous study. ${ }^{16}$ After adapting to the testing chamber for 5 minutes, the mice were separately exposed to aerosols from PBS and methacholine (MCh, SigmaAldrich) at concentrations of $6.25,12.5$ and $25 \mathrm{mg} / \mathrm{mL}$ for 3 minutes. 
A

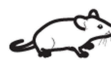

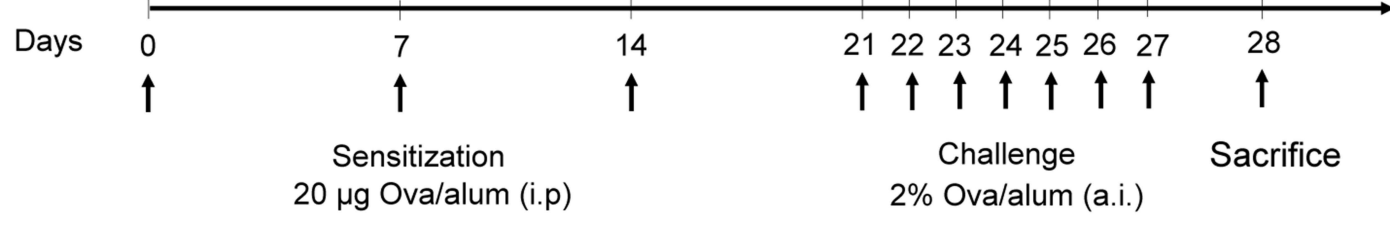

B

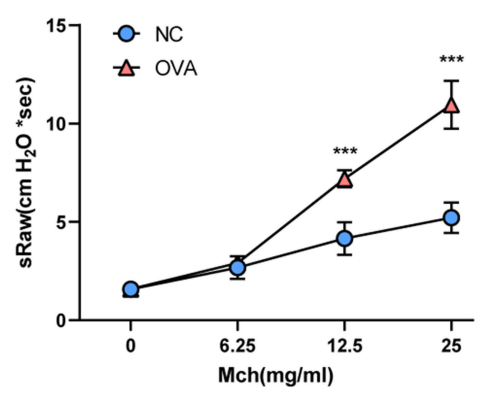

E

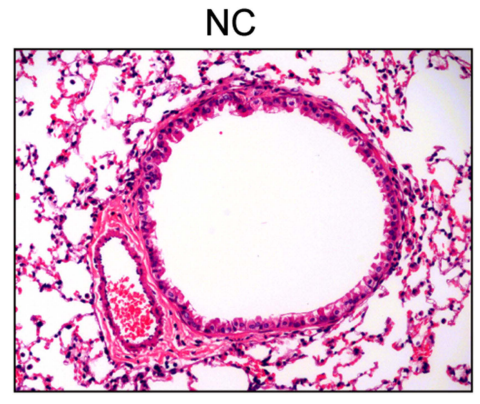

G

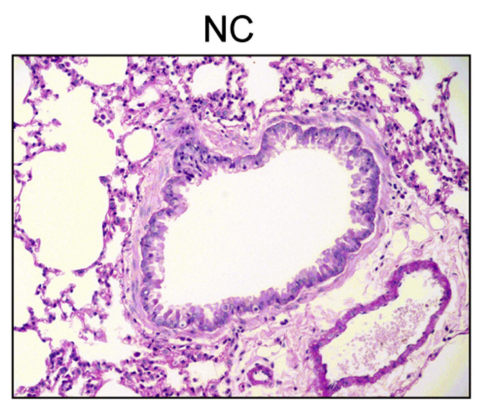

C
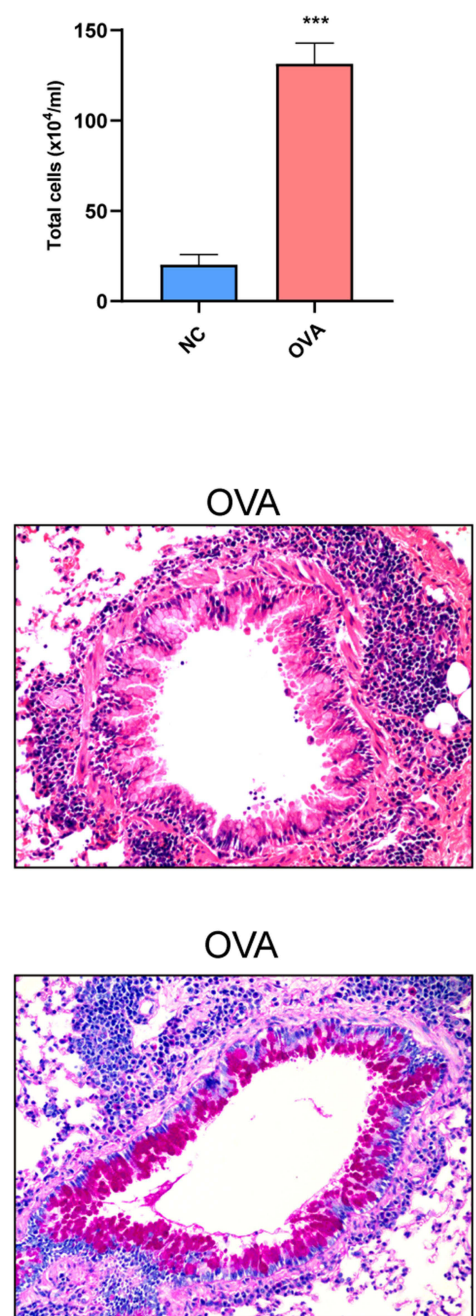

D

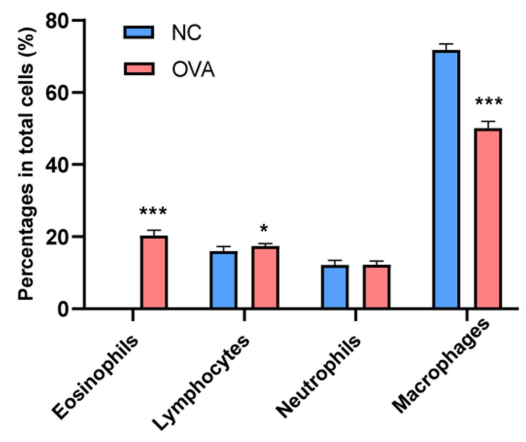

F

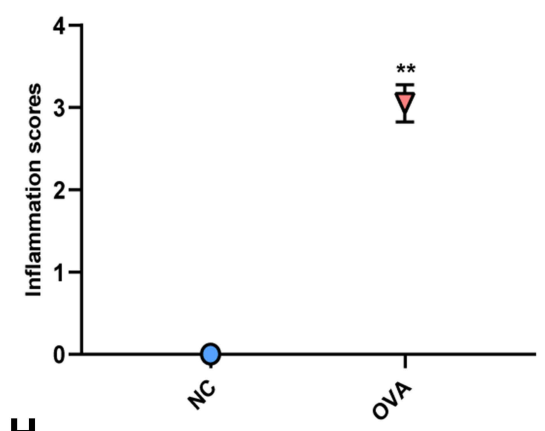

H

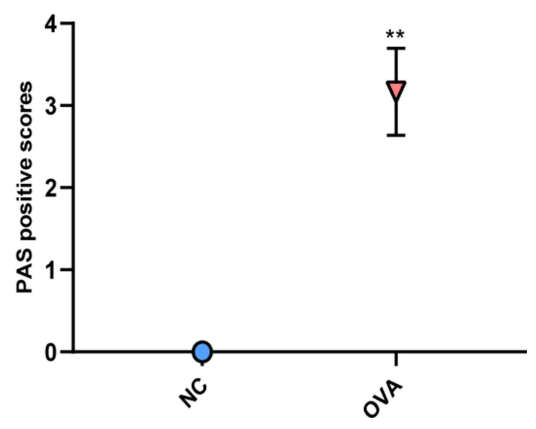

Figure I Airway inflammation and AHR of OVA-induced allergic asthma mice. (A) Experimental protocol. (B) Airway resistance to increased doses of Mch (mg/mL), presented as sRaw. (C) Total inflammatory cell count in BALF. (D) The mean percentage of eosinophils, lymphocytes, neutrophils and macrophages in BALF. (E) Representative HE-stained pictures of lung. (F) Inflammation scores. (G) Representative PAS-stained pictures of lung. (H) PAS-positive scores. Randomly selected three areas of each sample were used for semi-quantification. sRaw: specific airway resistance. (E and $\mathbf{G})$ Magnification, $\times 200$. Data are represented as expressed as mean \pm SD $(n=6) . * p<0.05, * * p<0.01$, and $* * * p<0.001$ versus the normal control group.

Abbreviations: Mch, Methacholine; BALF, Bronchoalveolar lavage fluid cell; NC, normal control group; OVA, asthma model group. 


\section{Bronchoalveolar Lavage Fluid Differential Cell Counts}

Bronchoalveolar lavage fluid (BALF) was collected after recording of sRaw, as previously described. ${ }^{15}$ Lungs of the mice were lavaged with $0.5 \mathrm{~mL}$ PBS solution for three times, and BALF was collected. The BALF was centrifuged at $1000 \mathrm{rpm}$ for 10 minutes at $4^{\circ} \mathrm{C}$. The separated cells were resuspended in PBS solution and counted with a hemocytometer. Cell classification was performed using Wright-Giemsa staining (Solarbio, Beijing, China), and a minimum of 300 cells were counted under optical microscopy.

\section{Histology}

The mice were sacrificed, 36 hours after the last OVA challenge, and the lung tissues were then collected for analysis. The lobes of left lungs were fixed with $4 \%$ paraformaldehyde, embedded in paraffin and sectioned into $3-\mu \mathrm{m}$-thick slices. Hematoxylin and eosin (HE) staining was used to evaluate inflammatory cell infiltration, the severity of peribronchial inflammatory cell infiltration was semi quantitatively scored as follows: score 0 means no inflammatory cell; score 1 means a few inflammatory cells; score 2 means a ring of inflammatory cells 1 cell layer deep; score 3 means a ring of inflammatory cells $2-4$ cells deep; and score 4 means a ring of inflammatory cells $>4$ cells deep. ${ }^{17}$

Periodic Acid-Schiff (PAS) staining was used to determine goblet cell hyperplasia and mucus secretion. The goblet cell (PAS-positive epithelial cell) hyperplasia in the airways was semi quantitatively scored as follows: score 0 means no goblet cells; score 1 means $<25 \%$ of the goblet cells; score 2 means between $25 \%$ and $50 \%$ of the goblet cells; score 3 means between $50 \%$ and $75 \%$ of the goblet cells; and score 4 means $>75 \%$ of the goblet cells. ${ }^{17}$ Histology scoring was determined under a microscope using two independent observers in a blinded manner.

\section{Real-Time PCR of Lung Tissues}

Total RNA extraction, reverse transcription and cDNA amplification were performed with the corresponding RNA simple Total RNA Kit (TIANGEN, Beijing, China), reverse transcription kit and SYBR-Green Premix (Takara, Otsu, Japan). Real-time PCR reactions were determined using a 7500 Real-Time PCR System (Applied Biosystems). Levels of mRNA expression were analyzed through the $2^{-\Delta \Delta C T}$ method. All the primer sequences shown in Table 1 were designed and synthesized by TsingKe Biological Technology Company (Beijing, China).

Table I Primer Sequences in RT-qPCR

\begin{tabular}{|l|l|}
\hline Gene Name & Primer Sequence (5' $\left.\rightarrow \mathbf{3}^{\prime}\right)$ \\
\hline Mouse Yapl & $\begin{array}{l}\text { F: GAGAAGGAGAGACTGCGGTTGAAAC } \\
\text { R: CCCTCCATCCTGCTCCAGTGTAG }\end{array}$ \\
\hline Mouse LefI & $\begin{array}{l}\text { F: CACACAACTGGCATCCCTCATCC } \\
\text { R: TGGGCTCCTGCTCCTTTCTCTG }\end{array}$ \\
\hline Mouse Ctgf & $\begin{array}{l}\text { F: AGAACTGTGTACGGAGCGTG } \\
\text { R: GTGCACCATCTTTGGCAGTG }\end{array}$ \\
\hline Mouse $\beta$-actin & $\begin{array}{l}\text { F: GGAGATTACTGCTCTGGCTCC } \\
\text { R: GCAGCTCAGTAACAGTCCG }\end{array}$ \\
\hline Human Wnt3a & $\begin{array}{l}\text { F: TGGGATGGTGTCTCGGGAGTTC } \\
\text { R: TCGTTGTTGTGGCGGTTCATGG }\end{array}$ \\
\hline Human Pard6b & $\begin{array}{l}\text { F: TCTGGCAGTTCCGGTCAGTCTAC } \\
\text { R: CTTCTTCGCTGTCTTCATCCTCTGG }\end{array}$ \\
\hline Human Wwcl & $\begin{array}{l}\text { F: GGACGCTGTGTCTGCTCTGTTG } \\
\text { R: CTCCTCCTCTTCCTCGGCTACTG }\end{array}$ \\
\hline Human $\beta$-actin & $\begin{array}{l}\text { F: CTCACCATGGATGATGATATCGC } \\
\text { R: CACATAGGAATCCTTCTGACCCA }\end{array}$ \\
\hline
\end{tabular}




\section{Double Immunofluorescence Staining}

To examine the relation between F4/80 expression and Yap1/Lef1/Ctgf expression in lung tissue, double immunofluorescence staining was performed. The F4/80 (1:5000, \# GB113373), Yap1 (1:200, \#GB11542), Lef1 (1:200, \# GB111887), Ctgf (1:200, \#GB11078) were purchased from Servicebio (Wuhan, China). The paraffin sections (3- $\mu \mathrm{m}$ thick) were dewaxed, rehydrated and blocked with $0.3 \% \mathrm{H}_{2} \mathrm{O}_{2}$, antigen repaired, as well as $10 \%$ goat serum closed. Slides were the incubated overnight with primary F4/80, Yap1, Lef1 and Ctgf antibodies, followed by incubation with the Alexa Fluor-488 (1:400, GB25303) and Cy3 conjugated Goat Anti-Rabbit IgG (H+L) (1:500, GB21303) secondary antibodies (servicebio, Wuhan, Hubei, China) for $50 \mathrm{~min}$. Finally, nuclei were stained with DAPI. The result was observed under a NIKON ECLIPSE C1 fluorescence microscopy (Nikon, Tokyo, Japan) and analyzed by image J software (NIH).

\section{Cell Treatment}

The human bronchial epithelial cell-line BEAS-2B was obtained from EK-Bioscience Company (Shanghai, China). IL13 is independent of other Th2 cytokines and is necessary and sufficient for all the characteristics of allergic asthma. ${ }^{18}$ In addition, IL-13 has critical roles in epithelial cell mucin production. ${ }^{19}$ BEAS-2B cells were divided into two groups: normal control group (NC group) and IL-13-stimulated group (IL-13 group). After the BEAS-2B cells were digested and seeded on culture plates, $20 \mathrm{ng} / \mathrm{mL}$ recombinant human IL-13 (\# 200-13, Peprotech) was added to the medium and incubated for 48 hours. The cells were collected for real-time PCR.

\section{RNA-Seq Analysis}

Lung tissues were collected from the lungs of the 3 mice of each group and pooled for mRNA sequencing. One $\mu \mathrm{g}$ of RNA per sample was used as input material for the RNA sample preparations. Sequencing libraries were generated using NEBNext UltraTM RNA Library Prep Kit for Illumina (NEB, USA). The library preparations were sequenced on an Illumina platform and paired-end reads were generated. The raw reads were further processed with an online platform bioinformatic pipeline tool, BMKCloud (www.biocloud.net).

Differential expression analysis was performed using the R package DESeq2 with a p-value cutoff of $0.05 .{ }^{20}$ The KEGG gene set enrichment analysis (GSEA) was performed using clusterProfiler. ${ }^{21}$ The protein-protein interaction (PPI) network of the DEGs was constructed through the STRING website (https://string-db.org/) and visualized using Cytoscape 3.7.2. Immune cell relative proportions from RNA-Seq data were calculated using the seq-ImmuCC (http://218.4.234.74:3200/immune/). The RNA-sequencing data from this study was uploaded to the NCBI Sequence Read Archive (SRA) and is available under Project ID PRJNA789695 (https://www.ncbi.nlm.nih.gov/sra/ PRJNA789695).

\section{External Datasets Validation}

The GSE143303 and GSE147878 datasets were obtained from the GEO (https://www.ncbi.nlm.nih.gov/geo/) and analyzed through GEO2R online. The data was explored using the website (www.lungcellatlas.org). Single-cell transcriptomics results of human bronchial biopsy in health and asthma were obtained from the study by Braga et $\mathrm{al}^{22}$ and analyzed using the website (www.lungcellatlas.org).

\section{Statistical Analysis}

The data obtained from this study were presented as means $\pm \mathrm{SD}$. One-way ANOVA analysis was used to evaluate the differences between the data in the different groups. The semi-quantitative data of the histopathological parameters were analyzed using the Kruskal-Wallis nonparametric test. All the data were analyzed using SPSS version 22.0 software (USA). The $P<0.05$ was considered to indicate statistical significance. ${ }^{*} P<0.05, * * P<0.01$ and ${ }^{* * *} P<0.001$ versus the NC group. 


\section{Results}

Airway Hyper-Responsiveness and Airway Inflammation in Mice with OVA-Induced Allergic Asthma

The process of OVA sensitization and asthma challenge is as shown in Figure 1A. Specific airway resistance (sRaw) was measured in response to increasing doses of nebulized methacholine. The airway responsiveness of the OVA group was significantly higher than that of NC group when mice were subjected to aerosols of Mch at concentrations of 12.5 and $25 \mathrm{mg} / \mathrm{mL}$. (Figure 1B). Bronchoalveolar lavage fluid (BALF) cells were identified under optical microscopy and differentiated into eosinophils, lymphocytes, neutrophils and macrophages. The total number of cells and the percentages of eosinophils were significantly higher in the OVA group than in the NC group (Figure 1C and D). The HE staining analysis showed that there was a significant infiltration of inflammatory cells around the airways in the OVA group (Figure 1E and F). On the other hand, the PAS staining analysis showed that there was a significant goblet cell hyperplasia and mucus hyperproduction around airways in the OVA group (Figure $1 \mathrm{G}$ and $\mathrm{H}$ ). These results revealed that the OVA-induced allergic asthma in mice was successfully established.

\section{RNA-Seq in Lung Tissue Identifies Hippo Signaling Pathway}

The raw reads counts were downloaded, and the read counts (RPKM) values from RNA-sequencing (RNA-seq) data were normalized (Additional file 1). Differential expression analysis (NC group VS OVA group) was performed using $\mathrm{R}$ package DESeq $2^{23}$ and a total of 6423 significant differential genes were obtained $(\mathrm{p}<0.05)$. The KEGG gene set enrichment analysis (GSEA) was performed using clusterProfiler. ${ }^{21}$ Gene set enrichment analysis results showed that
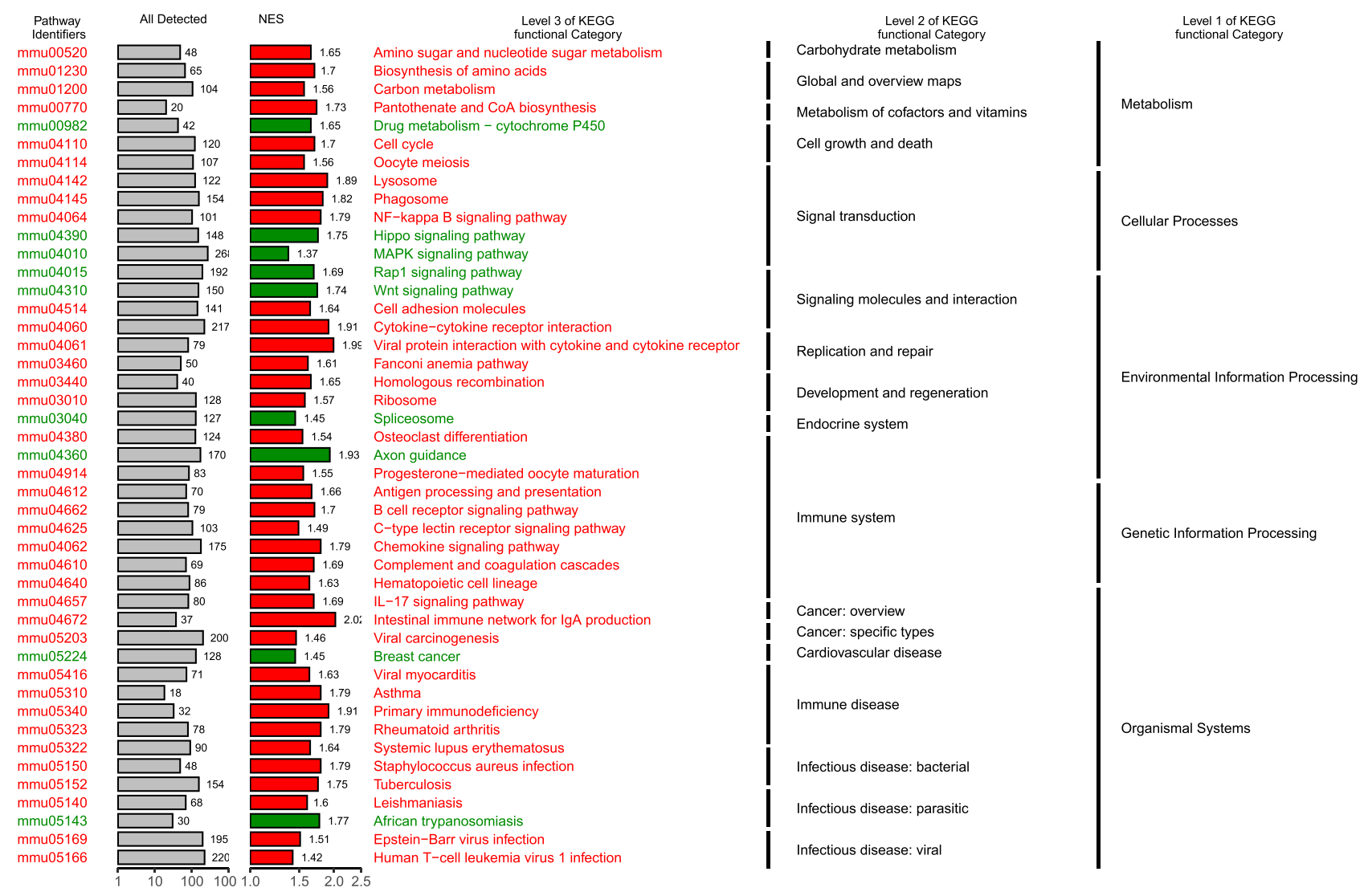

Figure 2 Gene set enrichment analysis (GSEA) enrichment map. Abbreviation: NES, normalized enrichment score. 
Hippo signaling pathway was significantly down-regulated in asthma mice model and is related to signal transduction in several cellular processes (Figure 2; Results are described in detail in Additional file 2).

\section{The Constructed Protein-Protein Interactional Network of DEGs and the Hippo Signaling Pathway Visualization}

A total of 59 genes were enriched into Hippo signaling pathway (Additional file 3). The protein-protein interaction (PPI) network of the DEGs was constructed using the STRING website (https://string-db.org/) and then visualized using Cytoscape 3.7.2 ${ }^{24}$ (Figure 3A). The top 10 hub genes by calculating the betweenness centrality were identified using cytoHubba tool kits in Cytoscape (Yap1, Lef1, Ctgf, Ccnd1, Axin2, Smad7, Wnt4, Wnt3a, Pard6b and Wwc1). It was found that the top scoring hub gene was Yap1, followed by Lef1 and Ctgf.

A

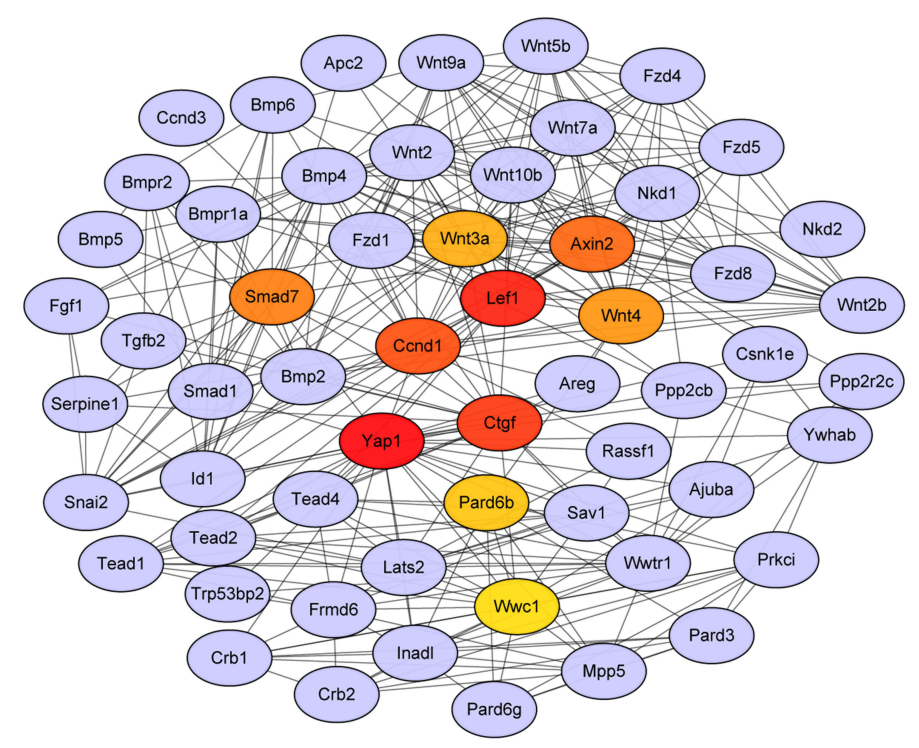

B

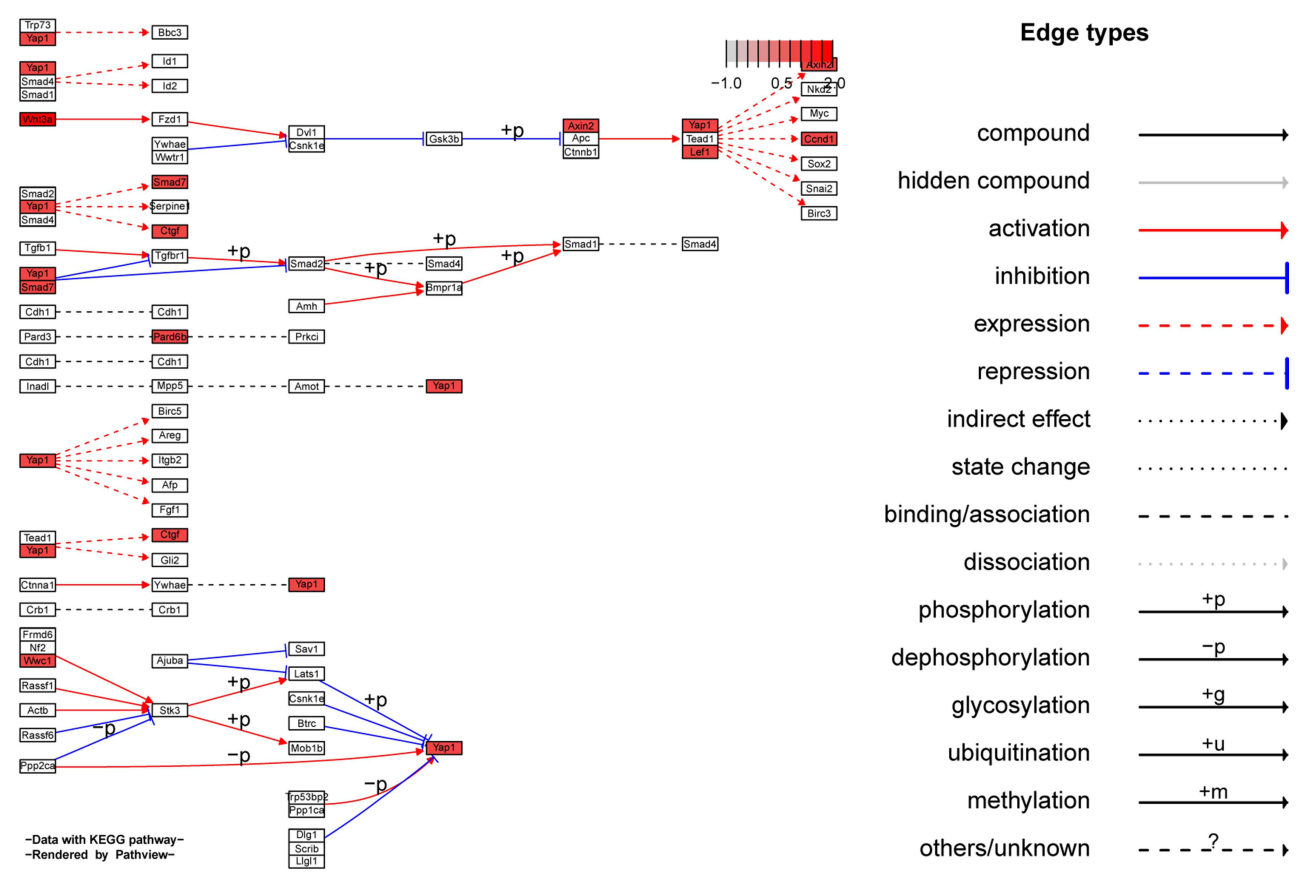

Figure 3 Hub genes of Hippo signaling pathway. (A) Network based on STRING and visualized in Cytoscape of the hub genes of the Hippo signaling pathway. (B) KEGG map of hub genes from Hippo signaling pathway rendered by Pathview. 
The top 10 hub genes were mapped onto KEGG pathways using the path-view package in $\mathrm{R}^{25}$ (Figure $3 \mathrm{~B}$ ). It is evident that Yap1 is a key regulator of Hippo signaling pathway and Yap1 exerts its functions through interaction with the functions of other hub genes, such as Lef1 and Ctgf (Figure 3B).

\section{Association Between Hippo Signaling Pathway and Macrophages}

Chen et $a^{26}$ developed a computational model named seq-ImmuCC to infer the relative proportions of 10 major immune cells in mouse tissues from RNA-Seq data. In the current study, immune cell relative proportions from RNA-Seq data in the lung tissue were calculated using the seq-ImmuCC (http://218.4.234.74:3200/immune/). Results of this study were as shown in Figure 4. Drastic changes in the number of macrophages and monocytes were seen in the NC and OVA groups. Noteworthy, the proportion of macrophages remarkably increased in OVA group compared with the NC group (Figure 4A). It was evident that the cell number of macrophages has a close relationship with allergic asthma. Combining our previous results as shown in Figures 2 and 3 (Hippo signaling pathway was significantly downregulated in a mouse model of asthma, 10 genes were selected as hub genes), it was conjectured that macrophages proliferation is associated with the downregulation of the Hippo signaling pathway.

In order to verify this conjecture, 10 hub genes were used as the representative geneset of the Hippo signaling pathway for single sample gene set enrichment analysis (ssGSEA). A correlational analysis between the 10 hub genes and macrophages cell proportions was then conducted using a 2-tailed Pearson test. Results of the analysis showed that the macrophages cell proportions and Hippo signaling pathway activity were strongly negatively correlated $\left(R^{2}=0.93\right.$; Figure $\left.4 B\right)$. In the present study, 10 hub genes were screened in the Hippo signaling pathway. It was found that the top 3 hub genes are Yap1, Lef1 and Ctgf. Therefore, the correlation between the macrophage proportions and RPKM values of Yap1, Lef1 and Ctgf was further evaluated. It was revealed that the results of the correlational analysis between macrophages proportions and RPKM values of Yap1 $\left(\mathrm{R}^{2}=0.89\right)$, Lef1 $\left(\mathrm{R}^{2}=0.87\right)$ and $\mathrm{Ctgf}\left(\mathrm{R}^{2}=0.78\right)$ were consistent (Figure 4C-E).

The relative mRNA expressions of Yap1, Lef1 and Ctgf were then measured in this study. The results showed that the relative mRNA expression of Yap1, Lef1 and Ctgf in the OVA group was significantly lower than that in the NC group (Figure 4F-H). This outcome further proved that the Hippo signaling pathway was significantly down-regulated in mice model of asthma. To determine whether the macrophages proliferation is related to the downregulation of the Hippo signaling pathway, a double immunofluorescence staining was performed to determine the coexpression of macrophages and Yap1/Lef1/Ctgf. Macrophages were identified with F4/80. The protein expression of F4/80 (labelled in red) and Yap1/Lef1/Ctgf (labelled in green) as well as their colocalization, as detected by double immunofluorescence (200×). The percentage of F4/80 positive cells was higher in the OVA group as compared with the NC group. Further, it was found that the percentage of Yap1/Lef1/Ctgf positive cells was higher in the NC group as compared with the OVA group. Double-stained immunofluorescence for F4/80 and Yap1, Lef1, Ctgf in mice lung sections 36 hours after the last OVA challenge, revealed that the macrophage proliferation is related to the downregulation of Hippo signaling pathway in OVA-induced allergic asthma mice (Figure 4I; magnification, $\times 200$ ). Semiquantification of F4/80 and Yap1, Lef1, Ctgf showed consistent results (Figure 4J-L). These data validate the conjecture that macrophages proliferation is associated with the downregulation of the Hippo signaling pathway.

\section{Validation Datasets of Hippo Signaling Pathway Downregulation in Asthma}

To assess whether our results in mice may be applied to human asthma, validation was performed using the GEO datasets. The GSE143303 (Transcriptome sequencing data of endobronchial biopsy samples from 47 asthma patients and 13 healthy people) and GSE147878 (Transcriptome sequencing data of endobronchial biopsy samples from 60 asthma patients and 13 healthy people) datasets were obtained from the GEO. Further, differential gene expression was calculated using GEO2R (https://www.ncbi.nlm.nih.gov/geo/geo2r/). The most downregulated top 500 genes were selected and KEGG pathway enrichment analysis was carried out using KOBAS-i ${ }^{27}$ (http://kobas.cbi.pku.edu.cn/), results are as shown in Figure 5. Similarly, it was found that Hippo signaling pathway was down-regulated in both datasets. This study validated the obtained results in 2 independent external validation datasets. 
A

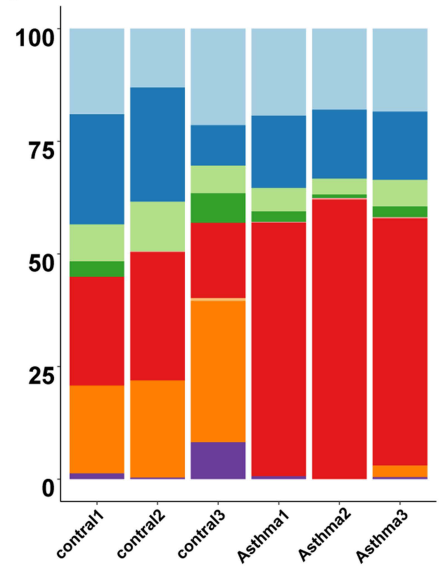

F

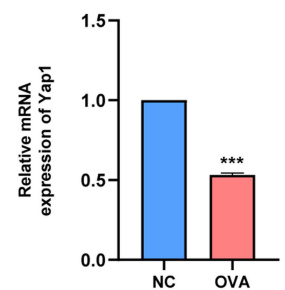

I

NC
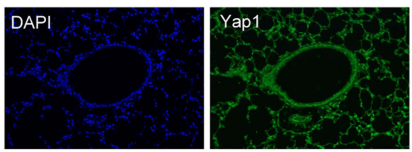

OVA
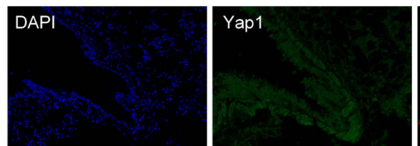

NC

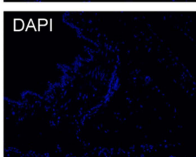

OVA
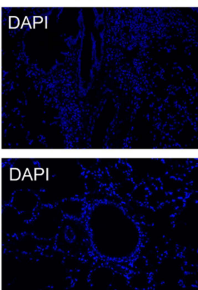

NC

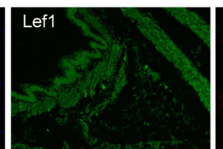

G
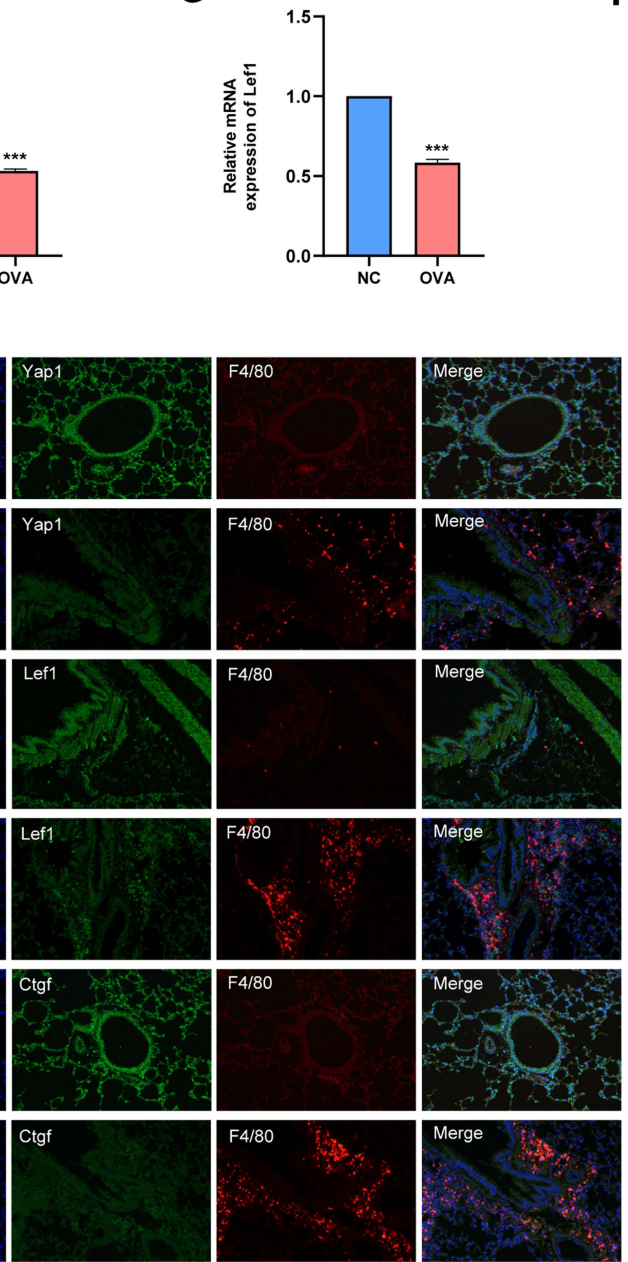
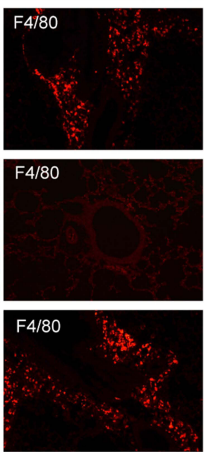

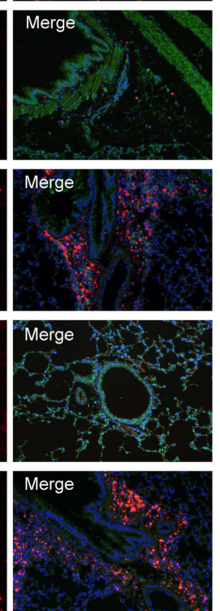

H

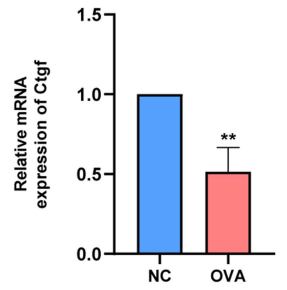

J

E
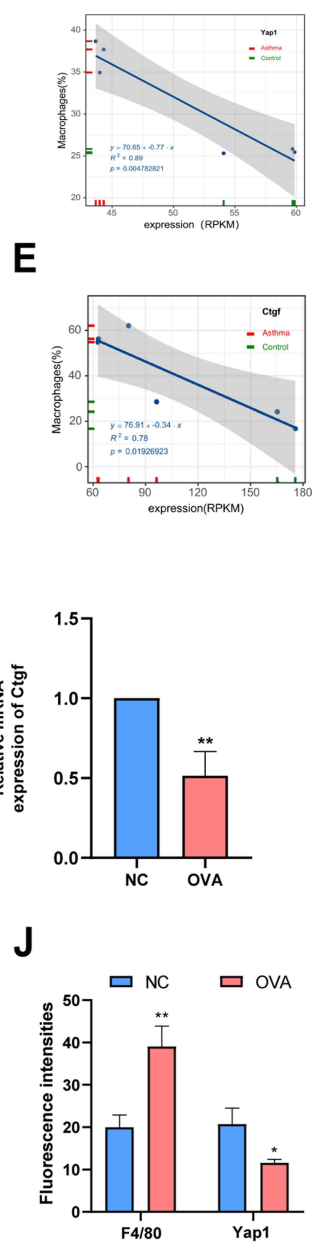

$\mathrm{K}$

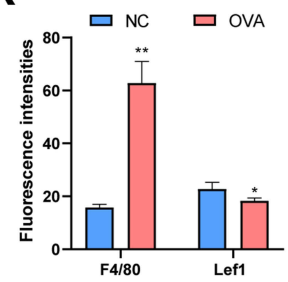

L

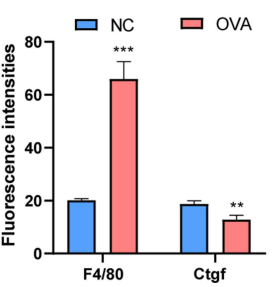

Figure 4 Macrophage proliferation is related to the downregulation of Hippo signaling pathway in OVA-induced allergic asthma mice. (A) Relationship between Hippo signaling pathway and macrophage in mice. Cellular compositions of immune microenvironment from Mouse RNA-seq data measured using the ImmuCC tool (http://2I8.4. 234.74:3200/immune/). (B) Correlation between ssGSEA scores of the 10 hub genes as Hippo signaling pathway gene sets and the proportion of macrophage obtained from plots (A) was analyzed. (C) Correlation between RPKM values of Yapl and the proportion of macrophage obtained from plots (A) was analyzed. (D) Correlation between RPKM values of Lefl and the proportion of macrophage obtained from plots (A) was analyzed. (E) Correlation between RPKM values of Ctgf and the proportion of macrophage obtained from plots (A) was analyzed. (F) Relative mRNA expression of Yapl. (G) Relative mRNA expression of Lefl. (H) Relative mRNA expression of Ctgf. (I) Double-stained immunofluorescence for F4/80 and YapI, Lefl, Ctgf in mouse lung sections 36 hours after OVA challenge respectively. (Magnification, $\times 200$ ) (J) Semiquantification of F4/80 and Yapl. (K) Semi-quantification of F4/80 and Lefl. (L) Semi-quantification of F4/80 and Ctgf. Randomly selected three areas of each sample were used for semi-quantification. Macrophages were identified with F4/80. Data are represented as expressed as mean $\pm S D(n=3)$. ${ }^{*}<<0.05$, $* * p<0.0 I$ and $* * * p<0.00 I$ versus the normal control group. Control I, Control 2 and Control 3: three from normal control mice were selected for lung tissue mRNA sequencing analysis. Asthma I, Asthma 2 and Asthma 3: three from normal control mice were selected for lung tissue mRNA sequencing analysis.

Abbreviations: NC, normal control group; OVA, asthma model group. 
A

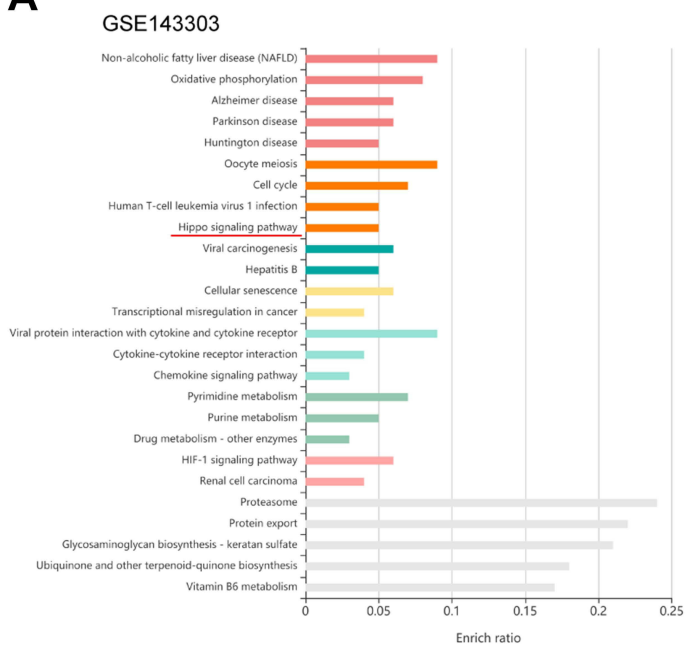

$\mathrm{C}_{1}$
$\mathrm{C} 2$
$\mathrm{C} 3$
$\mathrm{C}$
$\mathrm{C} 4$
$\mathrm{C} 5$
$\mathrm{C} 5$
$\mathrm{C}$
$\mathrm{C}$
$\mathrm{C}$
Other

C

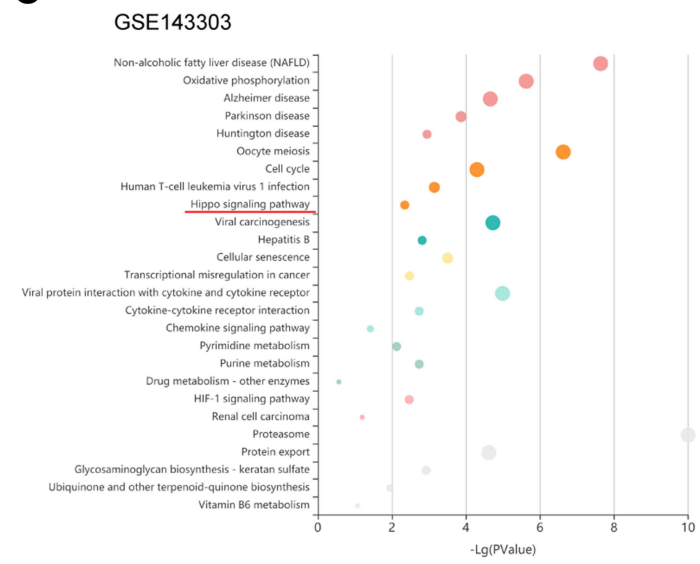

B

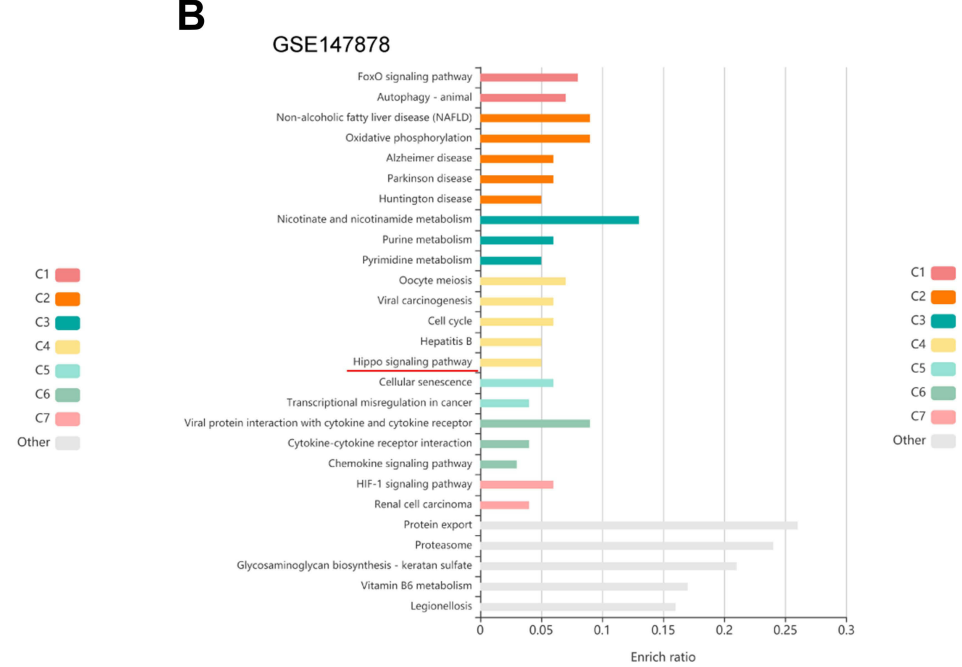

D

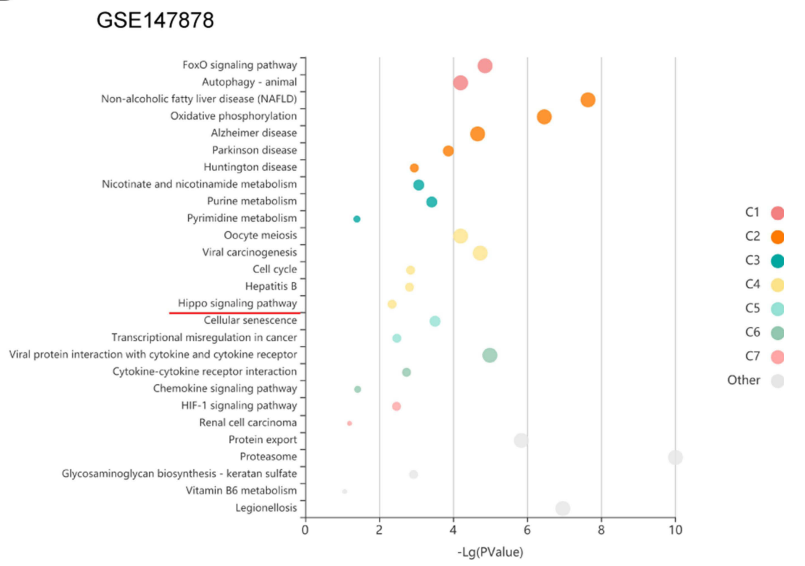

Figure 5 Validation of downregulation of the Hippo signaling pathway in endobronchial biopsies from asthma patients with GEO datasets. (A) Enrichment plots of Hippo signaling pathway; enriched in the GSEI43303. (B) Enrichment plots of Hippo signaling pathway; enriched in the GSEI47878. (C) Bubble plot shows -Log (P value) in the GSEI43303. (D) Bubble plot shows -Log (P value) in the GSEI47878.

\section{Hippo Signaling Pathway May Be Related to the Macrophages and Epithelial Cells Identified in the Human Asthmatic Bronchial Biopsies}

In a separate study by Braga et al, ${ }^{22}$ single-cell transcriptomics were used to complete the cellular census of upper and lower airways as well as the lung parenchyma in healthy lungs and lower airways in asthmatic lungs. The data was further explored and analyzed using the website (www.lungcellatlas.org). Moreover, the expression of 10 hub genes in bronchial biopsies of patients with asthma was also investigated. Results of this analysis showed that the expression of Ctgf, Ccnd1 and Axin2 is down-regulated in luminal macrophages (Figure 6A-F). Interestingly, the expression of 10 hub genes (Yap1, Lef1, Ctgf, Ccnd1, Axin2, Smad7, Wnt4, Wnt3a, Pard6b, and Wwc1) were down-regulated in airway epithelial cell such as goblet (Additional file 4). To validate whether Wnt3a, Pard6b, and Wwc1 is down-regulated in airway epithelial cell, this study compared the relative mRNA expression of BEAS-2B in the NC group and IL-13 group. It was found that that the relative mRNA expression levels of Wnt3a, Pard6b, and Wwc1 in the IL-13 group was significantly lower than that in the NC group (Figure 6G-O). These results illustrate that Hippo signaling pathway may be related to bronchial macrophages and epithelial cells. To summarize, the results show that the Hippo signaling pathway may be involved in the regulation of asthma by modulating macrophages and epithelial cells (Figure 7). 
A

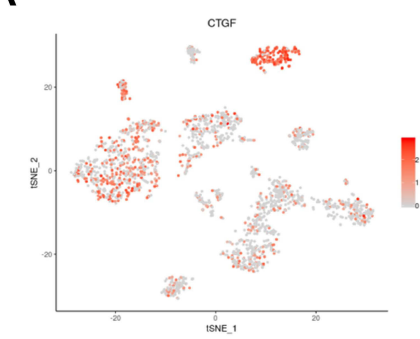

D

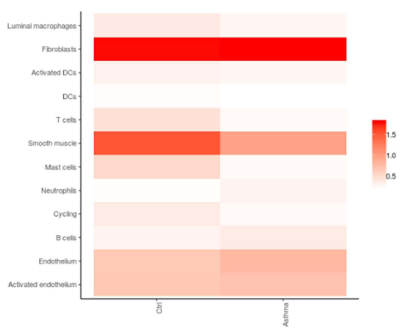

G

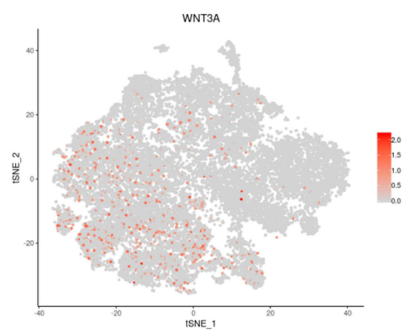

J

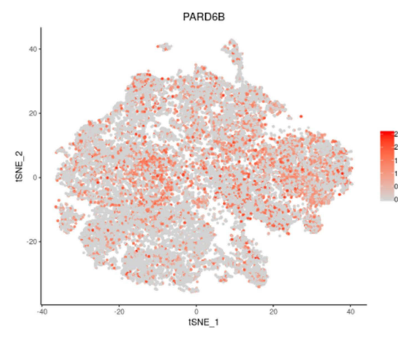

M

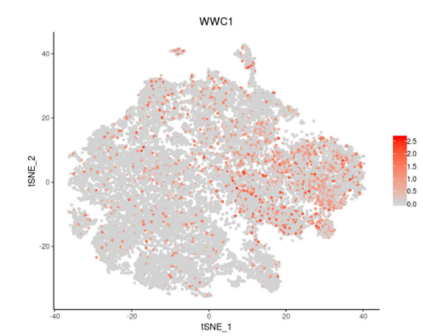

B

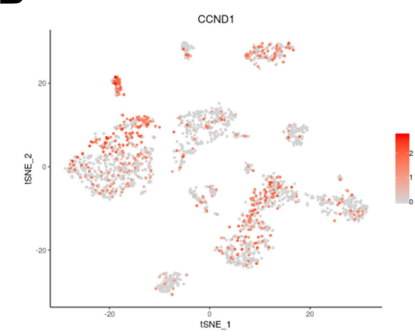

E

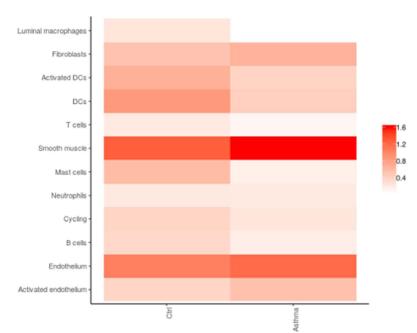

H

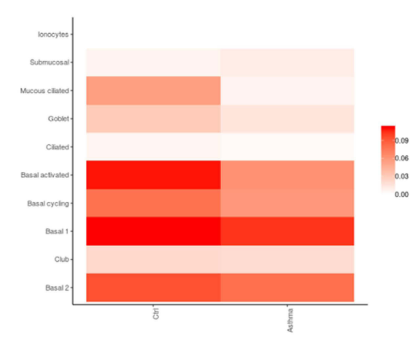

K

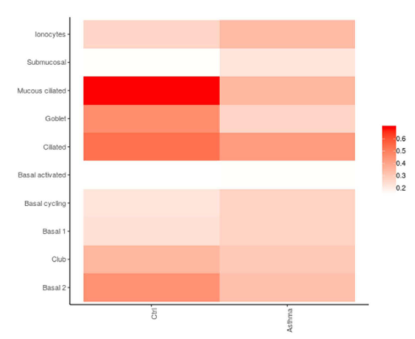

$\mathbf{N}$

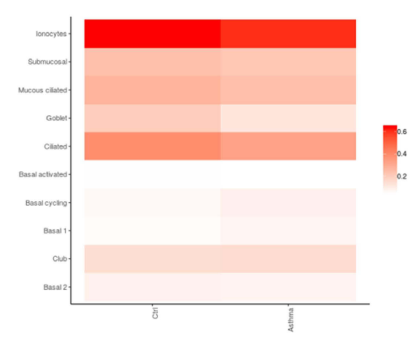

C

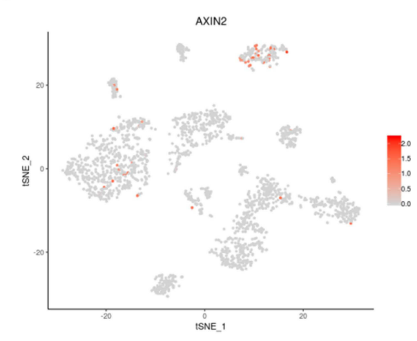

F

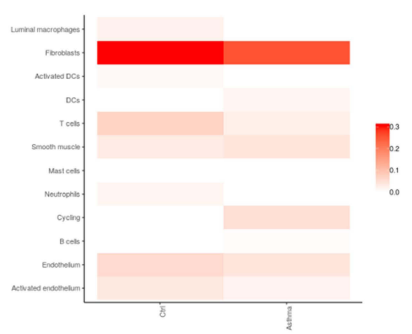

I

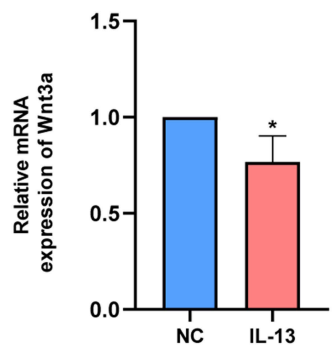

L

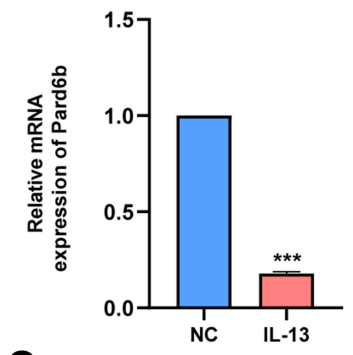

0

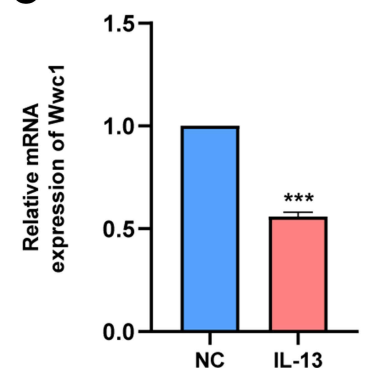

Figure 6 Hippo signaling pathway is related to the immune cell and epithelial cells identified in the human airway. (A) t-SNE displaying distribution of Ctof in airway. (B) t-SNE displaying distribution of Ccndl in airway. (C) t-SNE displaying distribution of Axin2 in airway. (D) Bar plot displaying distribution of Ctof in airway. (E) Bar plot displaying distribution of $\mathrm{Ccndl}$ in airway. (F) Bar plot displaying distribution of Axin2 in airway. (G) t-SNE displaying distribution of Wnt3a in airway epithelium. (H) Bar plot displaying distribution of Wnt3a in airway epithelium. (I) Relative mRNA expression of Wnt3a in BEAS-2B cells. (J) t-SNE displaying distribution of Pard6b in airway epithelium. (K) Bar plot displaying distribution of Pard6b in airway epithelium. (L) Relative mRNA expression of Pard6b in BEAS-2B cells. (M) t-SNE displaying distribution of Wwcl in airway epithelium. (N). Bar plot displaying distribution of Wwcl in airway epithelium. (O). Relative mRNA expression of Wwcl in BEAS-2B cells. NC: normal control group. IL-13: IL-13-stimulated group. Data are represented as expressed as mean $\pm S D(n=3)$. ${ }_{p} p<0.05$, and $* * * p<0.001$ versus the normal control group. Abbreviation: BEAS-2B, human bronchial epithelial cell line. 


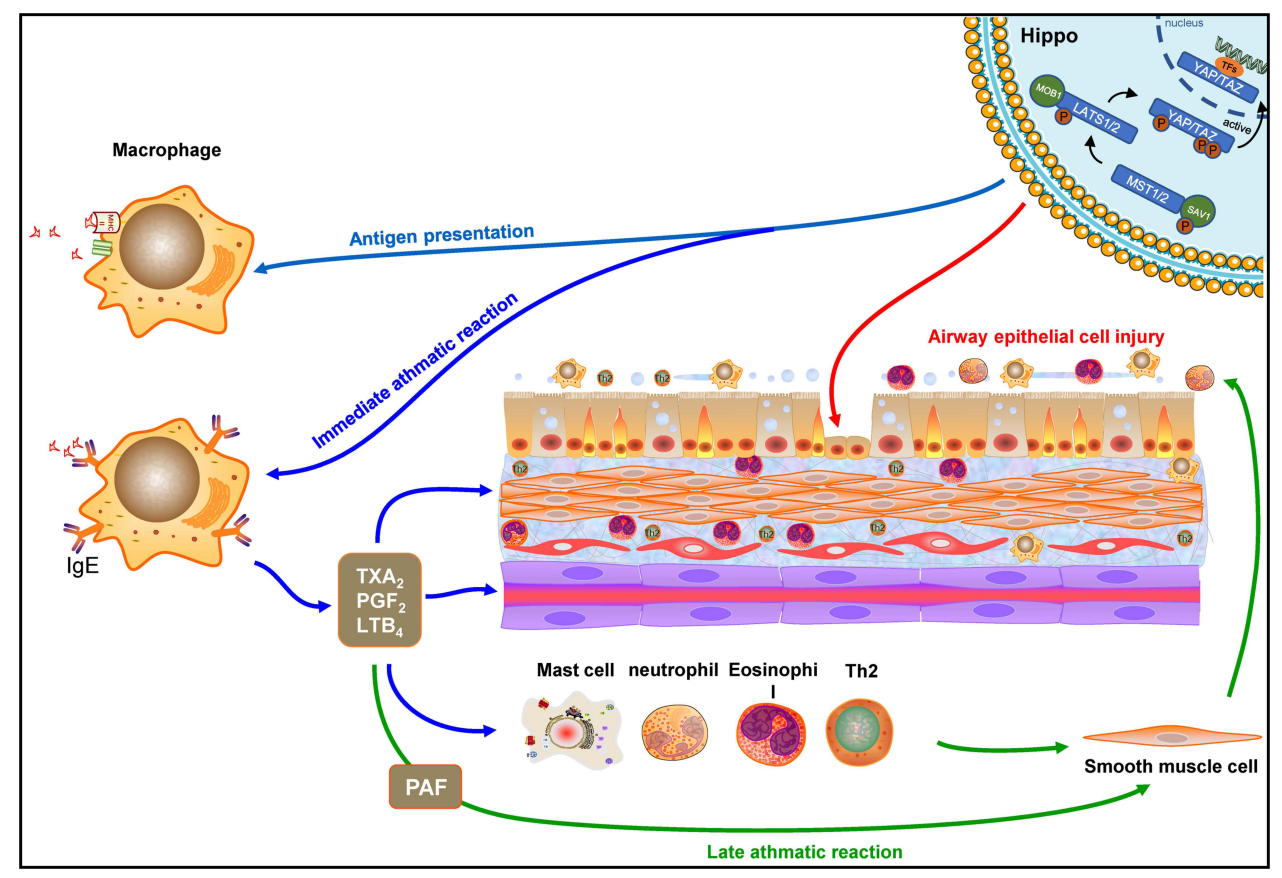

Figure 7 Diagram of the possible mechanisms between Hippo signaling pathway and macrophages of lung.

\section{Discussion}

In the present study, it was found for the first time that the proliferation of macrophages was significantly associated with down-regulation of Hippo signaling pathway in an allergic asthma mice model. The findings of the study were further validated on the RNA-sequencing dataset of human asthmatic patients. In addition, it was found that the regulation of epithelial cells in allergic asthma is also linked to Hippo signaling pathway. Therefore, the links between Hippo signaling pathway and macrophages in allergic asthma described in the present study provide novel insights into the pathogenesis and treatment of asthma.

Dysregulation of the Hippo signaling pathway has been implicated in many human diseases. ${ }^{28}$ In this study, it was found that Hippo signaling pathway was down-regulated in the lungs of mice with allergic-induced asthma. The findings were further validated on the GEO dataset of human asthmatic patients. Furthermore, the top 10 hub genes of Hippo signaling pathway that can regulate asthma were also screened out. It is evident that Yap1 is a major effector of Hippo signaling pathway. Furthermore, Ctgf and Ccnd1 are the two key downstream target genes of the YAP1-TEAD complex. Wwc1 is an upstream regulator of the Hippo signaling pathway and can regulate YAP activity through phosphorylation at serine 127. ${ }^{29}$ Pard6b as one of a Par complex component can be antagonized by Hippo signaling pathway. ${ }^{30}$ In addition, Lef1, Axin2, Wnt4 and Wnt3a, as Wnt signalling target genes, as well as Smad7, as TGF- $\beta$ inhibitor, are also important regulators of Hippo signaling pathways. These genes are mainly involved in the regulation of cell proliferation and have been reported to be involved in a variety of human cancers. ${ }^{31,32}$ Notably, recent studies have shown that the Hippo/YAP1 Pathway may play a role in asthma and allergy. Fu et $\mathrm{al}^{33}$ demonstrated that Fibulin-5 may promote proliferation and migration of airway smooth muscle cell (ASMC) in asthma by regulating Hippo-YAP/TAZ pathway. Moreover, Fodor et $\mathrm{al}^{34}$ have also suggested the FRMD6/Hippo/YAP1 pathway may have a function in asthma pathogenesis and its different subtypes. However, whether the other hub genes regulate asthma has not been reported. Therefore, the current study provides multiple new therapeutic targets of Hippo signaling pathway for allergic asthma.

Macrophage dysfunctions play an important role in asthma pathogenesis. These dysfunctions mainly include decreasing phagocytosis, efferocytosis and inflammatory resolution; increasing immune mediators and ROS production; and altered macrophage polarization. ${ }^{35}$ There are at least two types of tissue-resident macrophages in the lung, alveolar macrophages and interstitial macrophages. ${ }^{36}$ Recently, the role of alveolar macrophages in allergic asthma has gradually been revealed. It mainly focuses on three aspects: (1) antigen presentation function. (2) Involved in immediate asthmatic 
response. (3) Initiate and maintain a late asthmatic response. ${ }^{37}$ However, the molecular mechanisms responsible for macrophage dysfunctions still remain unclear. In this study, it was found that macrophages proliferation was significantly associated with the down-regulation of the Hippo signaling pathway. The results of the current study hence suggest that the downregulation of the Hippo signaling pathway may promote macrophages proliferation, which leads to asthma.

A study by Braga et $\mathrm{al}^{22}$ completed the cellular census of upper and lower airways and lung parenchyma in healthy lungs and lower airways in asthmatic lungs. Their research confirmed that macrophages are mainly distributed in lung parenchyma and are also distributed in bronchial biopsies. The expression of 10 hub genes of Hippo signaling pathway in their asthma airway atlas dataset was analyzed using the website (www.lungcellatlas.org). It was found that the expression of Ctgf, Ccnd1 and Axin2 is down-regulated in luminal macrophages in bronchial biopsies of patients with asthma. These results suggest that Hippo signaling pathway may be involved in the regulation of macrophages of bronchi.

Injury and shedding of airway epithelial cells are important histopathological features of asthma, which correlate with airway hyperresponsiveness and airway remodeling. ${ }^{38,39}$ The bronchial epithelium is predominantly composed of ciliated, goblet, basal, neuroendocrine, club cell, brush cell and Clara cells. ${ }^{40}$ Goblet cells play an important role in airway homeostasis by producing and secreting mucins. Overproduction and hypersecretion of mucin are hallmark features of asthma. ${ }^{41} \mathrm{~A}$ recent study showed that deletion of YAP/TAZ promotes goblet metaplasia and results in mucin hypersecretion in the lung epithelium of adult mice. ${ }^{42}$ These findings are similar to the findings obtained in the current study. Expressions of 10 hub genes of Hippo signaling pathway were also analyzed using the website (www.lungcellatlas.org) which was consistent with that in Braga et $\mathrm{al}^{22}$ asthma airway atlas epithelial dataset. That the expression of 10 hub genes (Yap1, Lef1, Ctgf, Ccnd1, Axin2, Smad7, Wnt4, Wnt3a, Pard6b, and Wwcl) is down-regulated in goblet cells in bronchial biopsies of patients with asthma suggest that Hippo signaling pathway may be also involved in the regulation of epithelial cells of bronchi.

It is thus suggested that Hippo signaling pathway may be involved in the regulation of macrophages and epithelial cells in asthma and may also play an important role in the pathogenesis of asthma.

Macrophages are an important drug target for asthma therapy. Currently, several therapies have already been used to treat asthma by modulating macrophage function, including corticosteroids, macrolides, statins, phosphodiesterase inhibitors (such as Theophylline, Roflumilast), leukotriene antagonists, $\beta$-Agonists. ${ }^{35}$ However, the role of macrophages in the pathogenesis of asthma is still not fully understood. Therefore, there is an urgent need for the development of new therapy target for asthma treatment. In this study, a negative regulatory relationship between macrophage proliferation and the Hippo signaling pathway was found and hence provided a new idea for the treatment of asthma with macrophages as the target. However, the specific regulation mechanism by which Hippo signaling pathway regulates the macrophage still requires further exploration.

\section{Conclusion}

In conclusion, the present study demonstrates that macrophages proliferation was significantly associated with the downregulation of the Hippo signaling pathway in asthma. Therefore, the current research demonstrated the Hippo signaling pathway as a potential target for the development of therapies against allergic asthma. However, there is a need for more studies to explore the specific regulation mechanism.

\section{Abbreviations}

Yap1, yes-associated protein 1; Lef1, lymphoid enhancer-binding factor 1; Ctgf, connective tissue growth factor; Ccnd1, cyclin D1; Axin2, axin 2; Smad7, SMAD family member 7; Wnt4, wingless-type MMTV integration site family, member 4; Wnt3a, wingless-type MMTV integration site family, member 3A; Pard6b, par-6 family cell polarity regulator beta; Wwc1, WW, C2 and coiled-coil domain containing 1; Taz, Tafazzin.

\section{Acknowledgment}

This study was supported by a grant from the Guangxi Natural Science Foundation (No. 2020GXNSFDA238003).

\section{Disclosure}

The authors report no conflicts of interest in this work. 


\section{References}

1. Fahy JV. Type 2 inflammation in asthma-present in most, absent in many. Nat Rev Immunol. 2015;15(1):57-65. doi:10.1038/nri3786

2. Jassal MS. Special considerations-asthma in children. Int Forum Allergy Rhinol. 2015;5(Suppl 1):S61-S67. doi:10.1002/alr.21577

3. Liu B, Zheng Y, Yin F, Yu J, Silverman N, Pan D. Toll receptor-mediated Hippo signaling controls innate immunity in drosophila. Cell. 2016;164 (3):406-419. doi:10.1016/j.cell.2015.12.029

4. Rauskolb C, Sun S, Sun G, Pan Y, Irvine KD. Cytoskeletal tension inhibits Hippo signaling through an Ajuba-Warts complex. Cell. 2014;158 (1):143-156. doi:10.1016/j.cell.2014.05.035

5. Meng Z, Moroishi T, Guan K-L. Mechanisms of Hippo pathway regulation. Genes Dev. 2016;30(1). doi:10.1101/gad.274027.115

6. Zhao B, Tumaneng K, Guan K-L. The Hippo pathway in organ size control, tissue regeneration and stem cell self-renewal. Nat Cell Biol. 2011;13 (8):877-883. doi:10.1038/ncb2303

7. Leach JP, Heallen T, Zhang M, et al. Hippo pathway deficiency reverses systolic heart failure after infarction. Nature. 2017;550(7675):260-264. doi:10.1038/nature24045

8. Moroishi T, Hayashi T, Pan -W-W, et al. The Hippo pathway kinases LATS1/2 suppress cancer immunity. Cell. 2016;167(6). doi:10.1016/j. cell.2016.11.005

9. Yu F-X, Zhao B, Guan K-L. Hippo pathway in organ size control, tissue homeostasis, and cancer. Cell. 2015;163(4):811-828. doi:10.1016/j. cell.2015.10.044

10. Nowell CS, Odermatt PD, Azzolin L, et al. Chronic inflammation imposes aberrant cell fate in regenerating epithelia through mechanotransduction. Nat Cell Biol. 2016;18(2):168-180. doi:10.1038/ncb3290

11. Zhang Y, Zhang H, Zhao B. Hippo signaling in the immune system. Trends Biochem Sci. 2018;43(2):77-80. doi:10.1016/j.tibs.2017.11.009

12. Umetsu DT, McIntire JJ, Akbari O, Macaubas C, DeKruyff RH. Asthma: an epidemic of dysregulated immunity. Nat Immunol. 2002;3(8):715-720. doi:10.1038/ni0802-715

13. Murray PJ, Allen JE, Biswas SK, et al. Macrophage activation and polarization: nomenclature and experimental guidelines. Immunity. 2014;41 (1):14-20. doi:10.1016/j.immuni.2014.06.008

14. Mooring M, Fowl BH, Lum SZC, et al. Hepatocyte stress increases expression of yes-associated protein and transcriptional coactivator with PDZ-binding Motif in hepatocytes to promote parenchymal inflammation and fibrosis. Hepatology. 2020;71(5):1813-1830. doi:10.1002/hep.30928

15. Zhang J, Li C, Guo S. Effects of inhaled inactivated mycobacterium phlei on airway inflammation in mouse asthmatic models. J Aerosol Med Pulm Drug Deliv. 2012;25(2). doi:10.1089/jamp.2011.0904

16. Jiang X-H, Li C-Q, Feng G-Y, Luo M-J, Sun Q-X. Inhalation of nebulized can protect against allergic bronchial asthma in mice by regulating the TGF- $\beta$ /Smad signal transduction pathway. Allergy Asthma Clin Immunol. 2020;16:59. doi:10.1186/s13223-020-00456-8

17. Fang L, Shen Q, Wu H, et al. TLR2 favors OVA-induced allergic airway inflammation in mice through JNK signaling pathway with activation of autophagy. Life Sci. 2020;256:117896. doi:10.1016/j.1fs.2020.117896

18. Wills-Karp M. Interleukin-13 in asthma pathogenesis. Immunol Rev. 2004;202:175-190. doi:10.1111/j.0105-2896.2004.00215.x

19. Zhen G, Park SW, Nguyenvu LT, et al. IL-13 and epidermal growth factor receptor have critical but distinct roles in epithelial cell mucin production. Am J Respir Cell Mol Biol. 2007;36(2):244-253. doi:10.1165/rcmb.2006-0180OC

20. Anders S, Huber W. Differential expression analysis for sequence count data. Genome Biol. 2010;11(10):R106. doi:10.1186/gb-2010-11-10-r106

21. Yu G, Wang L-G, Han Y, He Q-Y. clusterProfiler: an R package for comparing biological themes among gene clusters. OMICS. 2012;16 (5):284-287. doi:10.1089/omi.2011.0118

22. Braga FA, Kar G, Berg M, et al. A cellular census of human lungs identifies novel cell states in health and in asthma. Nat Med. 2019;25 (7):1153-1163. doi:10.1038/s41591-019-0468-5

23. Love MI, Huber W, Anders S. Moderated estimation of fold change and dispersion for RNA-seq data with DESeq2. Genome Biol. $2014 ; 15(12): 550$. doi:10.1186/s13059-014-0550-8

24. Shannon P, Markiel A, Ozier O, et al. Cytoscape: a software environment for integrated models of biomolecular interaction networks. Genome Res. 2003;13(11):2498-2504. doi:10.1101/gr.1239303

25. Luo W, Brouwer C. Pathview: an R/Bioconductor package for pathway-based data integration and visualization. Bioinformatics. 2013;29 (14):1830-1831. doi:10.1093/bioinformatics/btt285

26. Chen Z, Quan L, Huang A, et al. seq-ImmuCC: cell-centric view of tissue transcriptome measuring cellular compositions of immune microenvironment from mouse RNA-seq data. Front Immunol. 2018;9:1286. doi:10.3389/fimmu.2018.01286

27. Mao X, Cai T, Olyarchuk JG, Wei L. Automated genome annotation and pathway identification using the KEGG Orthology (KO) as a controlled vocabulary. Bioinformatics. 2005;21(19):3787-3793. doi:10.1093/bioinformatics/bti430

28. Plouffe SW, Hong AW, Guan K-L. Disease implications of the Hippo/YAP pathway. Trends Mol Med. 2015;21(4):212-222. doi:10.1016/j. molmed.2015.01.003

29. Saladi SV, Ross K, Karaayvaz M, et al. ACTL6A is co-amplified with p63 in squamous cell carcinoma to drive YAP activation, regenerative proliferation, and poor prognosis. Cancer Cell. 2017;31(1):35-49. doi:10.1016/j.ccell.2016.12.001

30. Frum T, Murphy TM, Ralston A. HIPPO signaling resolves embryonic cell fate conflicts during establishment of pluripotency in vivo. Elife. 2018;7:e42298

31. Cunliffe HE, Jiang Y, Fornace KM, Yang F, Meltzer PS. PAR6B is required for tight junction formation and activated PKC $\zeta$ localization in breast cancer. Am J Cancer Res. 2012;2(5):478-491.

32. Yu J, Zheng Y, Dong J, Klusza S, Deng W-M, Pan D. Kibra functions as a tumor suppressor protein that regulates Hippo signaling in conjunction with Merlin and expanded. Dev Cell. 2010;18(2):288-299. doi:10.1016/j.devcel.2009.12.012

33. Fu J, Zheng M, Zhang X, et al. Fibulin-5 promotes airway smooth muscle cell proliferation and migration via modulating Hippo-YAP/TAZ pathway. Biochem Biophys Res Commun. 2017;493(2):985-991. doi:10.1016/j.bbrc.2017.09.105

34. Fodor LE, Gézsi A, Ungvári L, et al. Investigation of the possible role of the Hippo/YAP1 pathway in asthma and allergy. Allergy Asthma Immunol Res. 2017;9(3):247-256. doi:10.4168/aair.2017.9.3.247

35. Fricker M, Gibson PG. Macrophage dysfunction in the pathogenesis and treatment of asthma. Eur Respir J. 2017;50(3). doi:10.1183/ 13993003.00196-2017 
36. Ginhoux F, Guilliams M. Tissue-resident macrophage ontogeny and homeostasis. Immunity. 2016;44(3):439-449. doi:10.1016/j.immuni.2016.02.024

37. Zhong NS, Xu J, Shi HZ, et al. Bronchial asthma: basic and clinical. In: Wang Q-Q, Cao X-T, editors. Antigen Presenting Cells. Beijing: People's Medical Publishing House; 2006:108-109.

38. Charokopos N, Apostolopoulos N, Kalapodi M, Leotsinidis M, Karamanos N, Mouzaki A. Bronchial asthma, chronic obstructive pulmonary disease and NF-kappaB. Curr Med Chem. 2009;16(7):867-883. doi:10.2174/092986709787549280

39. Bousquet J, Jeffery PK, Busse WW, Johnson M, Vignola AM. Asthma. From bronchoconstriction to airways inflammation and remodeling. Am J Respir Crit Care Med. 2000;161(5):1720-1745. doi:10.1164/ajrccm.161.5.9903102

40. Duclos GE, Teixeira VH, Autissier P, et al. Characterizing smoking-induced transcriptional heterogeneity in the human bronchial epithelium at single-cell resolution. Sci Adv. 2019;5(12):eaaw3413. doi:10.1126/sciadv.aaw3413

41. Fahy JV, Dickey BF. Airway mucus function and dysfunction. N Engl J Med. 2010;363(23):2233-2247. doi:10.1056/NEJMra0910061

42. Hicks-Berthet J, Ning B, Federico A, et al. Yap/Taz inhibit goblet cell fate to maintain lung epithelial homeostasis. Cell Rep. $2021 ; 36(2): 109347$. doi:10.1016/j.celrep.2021.109347

Journal of Inflammation Research

\section{Publish your work in this journal}

The Journal of Inflammation Research is an international, peer-reviewed open-access journal that welcomes laboratory and clinical findings on the molecular basis, cell biology and pharmacology of inflammation including original research, reviews, symposium reports, hypothesis formation and commentaries on: acute/chronic inflammation; mediators of inflammation; cellular processes; molecular mechanisms; pharmacology and novel anti-inflammatory drugs; clinical conditions involving inflammation. The manuscript management system is completely online and includes a very quick and fair peer-review system. Visit http://www.dovepress.com/testimonials.php to read real quotes from published authors.

Submit your manuscript here: https://www.dovepress.com/journal-of-inflammation-research-journal 